\title{
Control of the rat angiotensin I converting enzyme gene by CRE-like sequences
}

J. Xavier-Neto ${ }^{1}$, A.C. Pereira ${ }^{1}$, E.M. Oliveira ${ }^{2}$, A.A. Miyakawa ${ }^{1}$, M.L. Junqueira ${ }^{1}$ and J.E. Krieger ${ }^{1}$

\author{
${ }^{1}$ Laboratório de Genética e Cardiologia Molecular, Instituto do Coração, \\ Faculdade de Medicina, Universidade de São Paulo, São Paulo, SP, Brasil \\ ${ }^{2}$ Laboratório de Bioquímica da Atividade Motora, \\ Escola de Educação Física e Esporte, Universidade de São Paulo, \\ São Paulo, SP, Brasil
}

Correspondence

J.E. Krieger

Laboratório de Genética e

Cardiologia Molecular

InCor, FMRP, USP

Av. Dr. Enéas C. Aguiar, 44

05403-000 São Paulo, SP

Brasil

Fax: +55-11-3068-5048

E-mail: krieger@incor.usp.br

Research supported by FAPESP

(No. 01/00009-0).

Received July 14, 2003

Accepted June 8, 2004

\section{Abstract}

We characterized the role of potential cAMP-responsive elements (CRE) in basal and in induced angiotensin converting enzyme (ACE) gene promoter activity in order to shed light on the regulation of somatic ACE expression. We identified stimulators and repressors of basal expression between 122 and 288 bp and between 415 and 1303 bp upstream from the transcription start site, respectively, using a rabbit endothelial cell (REC) line. These regions also contained elements associated with the response to 8BrcAMP. When screening for CRE motifs we found pCRE, a proximal sequence between 209 and 222 bp. dCRE, a distal tandem of two CRE-like sequences conserved between rats, mice and humans, was detected between 834 and $846 \mathrm{bp}$. Gel retardation analysis of nuclear extracts of REC indicated that pCRE and dCRE bind to the same protein complexes as bound by a canonical CRE. Mutation of pCRE and dCRE in REC established the former as a positive element and the latter as a negative element. In 293 cells, a renal cell line, pCRE and dCRE are negative regulators. Co-transfection of ATF-2 or ATF-2 plus c-Jun repressed ACE promoter activity, suggesting that the ACE gene is controlled by cellular stress. Although mapping of cAMP responsiveness was consistent with roles for pCRE and dCRE, mutation analysis indicated that they were not required for cAMP responsiveness. We conclude that the basal activity of the somatic ACE promoter is controlled by proximal and distal CREs that can act as enhancers or repressors depending on the cell context.
Angiotensin converting enzyme (ACE) expression in cultured cells is modulated by hormones, ionophores, second messengers, and growth factors (1-5). The flexibility of modulation suggested by these properties supports the notion of regulated ACE ex-

\section{Introduction}

Key words

- Angiotensin converting enzyme

- Endothelium

- cAMP

- Cyclic AMP responsive

element 
useful to understand several pathological processes such as myocardial ischemia-reperfusion (6) and chronic inflammatory diseases such as sarcoidosis in which ACE is thought to play a role (7).

One of the most consistent stimulators of ACE activity and ACE mRNA expression is the cyclic AMP (cAMP)-dependent signaling pathway. cAMP analogues, adenylyl cyclase activators (forskolin) and agents acting through the beta adrenergic signal transduction pathway induce ACE activity and ACE mRNA expression in intact animals and in cell culture (8-10). Transduction of the intracellular cAMP signal is often performed by cAMP responsive element (CRE) genetic pathways. CRE pathways are activated upon phosphorylation of transcription factors bound to the prototypical CRE nucleotide sequence, TGACGTCA, or to its variants located in regulatory regions of target genes (11). Interestingly, non-canonical CREs mediate either cAMP responsiveness or regulation by distinct signaling pathways. In fact, CRE-like sequences often conserve the ability to bind transcription factors traditionally associated with cAMP signaling, while simultaneously binding other classes of transcription factors. Therefore, CRE-like sequences may confer multiple control possibilities to a regulatory region, integrating different signaling inputs $(12,13)$.

We have shown that increases in intracellular cAMP levels induced by stimulation of $B$-adrenergic receptors, activate transcription by the rat 1303-bp somatic ACE promoter in rabbit endothelial cells (REC) (10). Here we demonstrate that cAMP activates the CRE pathway but not the AP2 pathway, an alternative route for CAMP regulation. This suggests that CRE participates in the regulation of the ACE gene in endothelial cells. We performed mutational and gel retardation analysis of the 5' flanking region of the ACE gene to assess the role of CRE regulation in basal as well as in cAMP-induced activity of the ACE promoter. We report evidence that the basal activity of the ACE promoter in endothelial cells is controlled by both proximal and distal CRE-like sequences that display positive and negative regulatory activity, respectively. Interestingly, these CRElike sequences did not participate in the stimulation by $8 \mathrm{BrcAMP}$, indicating that the $\mathrm{ACE}$ gene uses a different set of regulators to respond to cAMP. Thus, our data indicate that the basal regulation of ACE promoter activity is complex and suggest that the ACE gene is a target for signal transduction pathways that use CRE-like sequences, but do not operate through the classic mechanisms utilized by cAMP.

\section{Material and Methods}

\section{Material}

Cell culture materials and media were from Gibco/Invitrogen (Carlsbad, CA, USA). 8BrcAMP was purchased from Sigma (St. Louis, MO, USA). Cell lysis buffers and the luciferase assay system were from Promega (Madison, WI, USA). The B-galactosidase and renilla luciferase assay systems were from Tropix/Perkin Elmer (Boston, MA, USA) and Promega, respectively.

\section{Plasmids and plasmid constructions}

The 1303-bp ACE promoter (-1274/+29), a positive control for CRE stimulation (CRETK) and a negative control for AP2 stimulation (TATALUC) have been described $(10,14)$. Deletion mutants covering wide regions of the 5' ACE region were synthesized by restriction enzyme digestion. The 122-bp ACE promoter was synthesized by a standard PCR. The PCR fragment was digested with Sac-I and Nhe-I and subcloned into the pGL2 basic luciferase vector (Promega). A negative control for cAMP stimulation, the thymidine kinase (TK) promoter construct, was prepared by removing 4 tandem repeats of CRE from pTKCAT4CRE 
(15) and also by subcloning pTKCAT4CRE into pGL2. We synthesized a series of hybrid ACE-TK constructs to identify potential cAMP responsive sequences between the 1303- and 228-bp nucleotides of the ACE promoter. A Kpn-I/Sac-I fragment containing ACE promoter sequences from 1281 to 228 bp was subcloned upstream from TK, generating the construct ACE1281-288-TK. Further deletion mutants, ACE1120-228-TK and ACE415-228-TK, were obtained by restriction enzyme digestion. The ACE845228-TK construct was synthesized by Pfu PCR. The PCR fragment was digested with Nhe-I and Bgl-II and subcloned into pGL2. A positive control for AP2 stimulation (AP2LUC) was constructed by subcloning a Sal-I/Bgl-II fragment from A2BCAT4 (16) containing three tandem repeats of a 19-bp hMtIIa AP2-binding site fused to the adenovirus E1b tata box, into pGL2. pact-CREBP1/ATF-2 (15) was a gift from Dr. Shunsuke Ishii, Laboratory of Molecular Genetics, RIKEN, Tsukuba, Ibaraki, Japan. The c-Jun and c-Fos expression vectors (17) were provided by Dr. Moshe Yaniv, Gene Expression and Disease Unit, Department of Developmental Biology, Pasteur Institute, Paris, France.

\section{Mutagenesis}

Mutant ACE promoters mdCRE, mpCRE and mdCREpCRE, each respectively harboring Bgl-II sites (AGATCT) in place of dCRE, pCRE or both, were synthesized using extension of mutated oligonucleotides mdCRE, 5' AGTGTGGAGACCAGATCTA GATCTAAGCCGATCTGTCTCAGG3', mpCRE AGCGAGAGCTCGACCAGATC TCATCCTTCCACCC by a Pfu PCR and subsequent digestion of the 1303 ACE promoter plasmid template by Dpn-I with a mutagenesis kit from Stratagene (La Jolla, CA, USA). Mutated constructs were selected by restriction analysis and confirmed by sequencing.

\section{Transient transfections}

REC cells (18) or human renal embryonic cells (293; Invitrogen) were grown in 12- or 24-well plates on F12 medium (F12 Coon's modification) or DMEM, respectively, and supplemented with $10 \%$ fetal bovine serum (FBS) and antibiotics (penicillin and streptomycin). Before reaching confluence, REC and 293 were transfected (16-24 h) with $3.25 \mu \mathrm{g}$ of luciferase reporter plasmids and $0.48 \mu \mathrm{g}$ of a control plasmid (pSVB-galactosidase; Promega) per well by the calcium phosphate method (19). Experiments employing expression vectors were performed with transfection of $1.78 \mu \mathrm{g}$ of luciferase reporter plasmids, $0.48 \mu \mathrm{g}$ of LTR$B$-galactosidase and $0.48 \mu \mathrm{g}$ of each expression vector per well. Thus, we used the same amounts of DNA for each expression vector when they were co-transfected. Total DNA was maintained at $3.73 \mu \mathrm{g}$ per well by manipulating the amount of pBluescript SK+ (Stratagene).

\section{cAMP treatment}

Prior to stimulation, transiently transfected cells were serum-starved for 16-24 h on F12 supplemented with $0.5 \%$ FBS. 8BrcAMP was then administered to cells at a concentration of $5 \mathrm{mM}$ for $4 \mathrm{~h}$ (10).

\section{Enzyme assays}

Luciferase and $\beta$-galactosidase were measured in cell extracts from transient transfections with a model 2010 Monolight luminometer, Analytical Luminescence Laboratory (San Diego, CA, USA) according to the manufacturer's instructions.

\section{Gel shift binding assay}

Nuclear proteins of REC were extracted according to established protocols (20). Binding of REC nuclear extracts to DNA was 
assayed by gel retardation analysis (20). The DNA used in these experiments was obtained by synthesis of complementary oligonucleotides representing the ACE promoter sequences of 796 to $776 \mathrm{bp}$ (TGTGGA GACCTGAGGTGACTTGAAG), 199 to 174 bp (GAGCTCGACCTAACCTCATCC TTCCA) and oligonucleotides containing the canonical CRE (AGAGATTGCCTGACGT CAGAGAGCT). Protein-DNA complexes were visualized by labeling DNA with ${ }^{32} \mathrm{P}$ by a filling-in reaction using Klenow polymerase.

\section{Sequence alignment and promoter}

Rat, mouse (M34433), rabbit (M58580), and human (M34434) ACE promoters were aligned with CLUSTAL W at http:// transfac.gbf.de/. Detection of transcription factor binding sites was performed with MatInspector public domain software at http:/ /genomatix.gsf.de/free_services/.

\section{Data presentation}

Luciferase activity in extracts of transiently transfected cells was normalized for ß-galactosidase activity or renilla luciferase in order to control for differences in transfection efficiency. Data are reported as means \pm SEM. Unless otherwise stated, data are reported as variation from control values. Where appropriate, results were analyzed by one-way analysis of variance. Statistical significance was set at $\mathrm{P}<0.05$.

\section{Results}

\section{ACE promoter regions associated with basal and CAMP-induced regulation}

To investigate the role of CRE-like sequences in basal and cAMP-induced regulation of the ACE gene we first determined promoter regions containing potential regulators and then screened these sites for the presence of CRE-like motifs.

We transfected REC cells with luciferase constructs representing a nested set of fragments from the 5 ' regulatory region of the rat somatic ACE gene. The fragments contained 122, 228, 415, and 1303 bp (21). Luciferase expression from the 122-bp promoter was the smallest. Significant increases in luciferase expression were detected with 228and 415-bp promoter fragments, suggesting the presence of positive regulatory sequences between 122 and $228 \mathrm{bp}$, as well as between 228 and 415 bp upstream of the transcription start site. Luciferase expression by the 1303bp promoter, however, was approximately half that by the 415-bp promoter, indicating the presence of negative sequences between 415 and 1303 bp upstream of the transcription start site (Figure 1). Luciferase expression from all ACE promoter constructs was on average 50 times higher than the promoterless pGL2 vector, but 50 times less than luciferase expression driven by the SV40 promoter (Figure 1, inset). Thus, deletion analyses of the 5 ' regulatory region of the ACE gene suggested the presence of positive regulators between 122 and $415 \mathrm{bp}$ and negative regulators between 415 and 1303 bp.

To find promoter regions associated with the response to cAMP induction we transfected REC cells with 122-, 228-, 415-, and 1303-bp ACE promoter fragments, as well as with positive (CRETK) and negative (TK) controls for activation of the CRE pathway. Induction of ACE promoter expression by 8BrcAMP was sequence-dependent. Stimulation of the 122-bp construct produced minimal increases in luciferase expression, similar to those of TK, the negative control. Induction of luciferase expression by 8 BrcAMP increased substantially by the 228bp promoter, but was not further increased by the $415-b p$ promoter. In contrast, the 1303-bp promoter displayed levels of luciferase induction by $8 \mathrm{BrcAMP}$ twice as high as those of the 228-bp promoter and of 
the same order of magnitude as those from CRETK, the positive control. Thus, deletion analysis of cAMP responsiveness suggested the presence of proximal CREs between 122 and $228 \mathrm{bp}$ as well as additional distal CREs between 415 and 1303 (Figure 2A).

To establish whether putative distal CREs between 415 and 1303 bp operate autonomously or only in association with the proximal CREs located between 122 and $228 \mathrm{bp}$, we subcloned ACE promoter fragments 228 to 415,228 to 845,228 to 1120 , and 228 to 1281 bp upstream from the heterologous TK promoter. Consistent with the above deletion analysis, addition of nucleotides 228 to 415 did not affect the cAMP responsiveness of the TK promoter. Remarkably, addition of nucleotides 228 to 845 doubled the induction of the TK promoter by 8 BrcAMP. However, further addition of nucleotides did not change cAMP responsiveness of hybrid ACETK promoters, indicating that distal sequences containing functional CREs must lie between 415 and 845 bp (Figure 2B). Thus, deletion analyses of distal ACE promoter regions in the context of the heterologous TK promoter indicated that there are CREs acting in an autonomous fashion between 415 and 845 bp.

\section{pCRE and dCRE are CRE-like sequences of the 1303-bp ACE promoter}

Inspection of the 1303-bp ACE promoter revealed the presence of two CRE-like sequences representing 2 bp substitutions from the canonical CRE, TGACGTCA. One proximal CRE-like sequence, TAACCTCA, (pCRE) was found between 209 and $222 \mathrm{bp}$ upstream from the transcription initiation. Another distal CRE-like sequence, TGAGG TGACTTGA, (dCRE) represented 2 tandem overlapping repeats located between 801 and $819 \mathrm{bp}$ upstream from the transcription initiation (Figure 3). Alignment of rat, mouse, rabbit, and human ACE 5 ' regulatory regions indicated that only dCRE is evolutionarily conserved in these three species.

As established by functional analysis, the position of pCRE is consistent with the location of a positive regulator of basal expression and with a functional CRE mediating induction by $8 \mathrm{BrcAMP}$. Likewise, the position of dCRE is consistent with the location of a negative regulator of basal transcription as well as a functional CRE mediating 8BrcAMP responsiveness.

\section{Gel retardation analysis of pCRE and dCRE}

To test the ability of pCRE and dCRE sequences to bind CRE-binding proteins we performed gel retardation assays. Figure 4 shows that REC nuclear extracts contain protein complexes that bind $\mathrm{pCRE}$ and $\mathrm{dCRE}$ (Figure 4A and B, respectively). Binding of these ACE CREs can be displaced by the

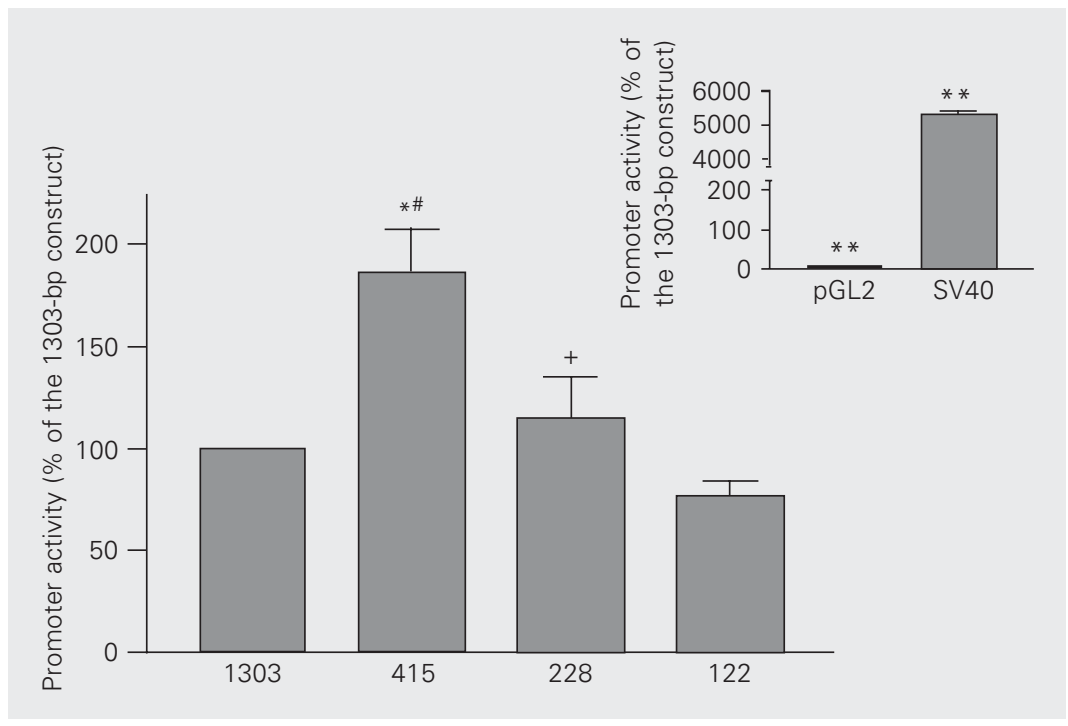

Figure 1. Mapping of elements that regulate the basal expression of the angiotensin converting enzyme (ACE) promoter in an endothelial cell line (REC). The wild type 1303-bp ACE promoter, three deletion mutants $(415,228$ and $122 \mathrm{bp}$ ) and controls for high (SV40) or low expression (pGL2) were transfected in REC to identify ACE promoter sequences with a role in basal expression. Cells were harvested $24 \mathrm{~h}$ after transfection. Luciferase activity in cell extracts was normalized for beta galactosidase expression from a control plasmid. Promoter activity is the ratio of luciferase/beta galactoside expression and is reported as percent of the activity of the 1303-bp ACE promoter. Data are reported as the mean \pm SEM for 4 independent experiments ( $N=4$ in each). Data for the reporter plasmids pGL2 and SV40 (inset) were obtained in the same experiments, but displayed separately to accommodate large variations in scale. ${ }^{*} \mathrm{P}<0.05$ compared to $1303 \mathrm{bp},{ }^{*} \mathrm{P}<0.0001$ compared to $1303 \mathrm{bp}, \# \mathrm{P}<0.05$ compared to $228 \mathrm{bp},+\mathrm{P}<0.05$ compared to $122 \mathrm{bp}$ (one-way analysis of variance and post hoc Tukey test). 
addition of excess unlabeled canonical CRE, indicating that $\mathrm{pCRE}$ and $\mathrm{dCRE}$ bind to CREbinding proteins in a reversible and competitive manner (Figure 4A,B). Interestingly, the protein complexes that bind to $\mathrm{dCRE}$ and pCRE (data for pCRE are not shown) appear to represent a subset of those binding the canonical CRE. ACE CRE-like motifs bind protein complexes with much lower affinity than the canonical CRE (Figure 4C), which is consistent with both $\mathrm{dCRE}$ and pCRE being 2 bp substitutions from the canonical CRE.

\section{Functional assessment of pCRE and dCRE in basal and cAMP-induced regulation of the ACE promoter}

To test the function of $\mathrm{pCRE}$ and $\mathrm{dCRE}$ in basal and cAMP-stimulated transcription,

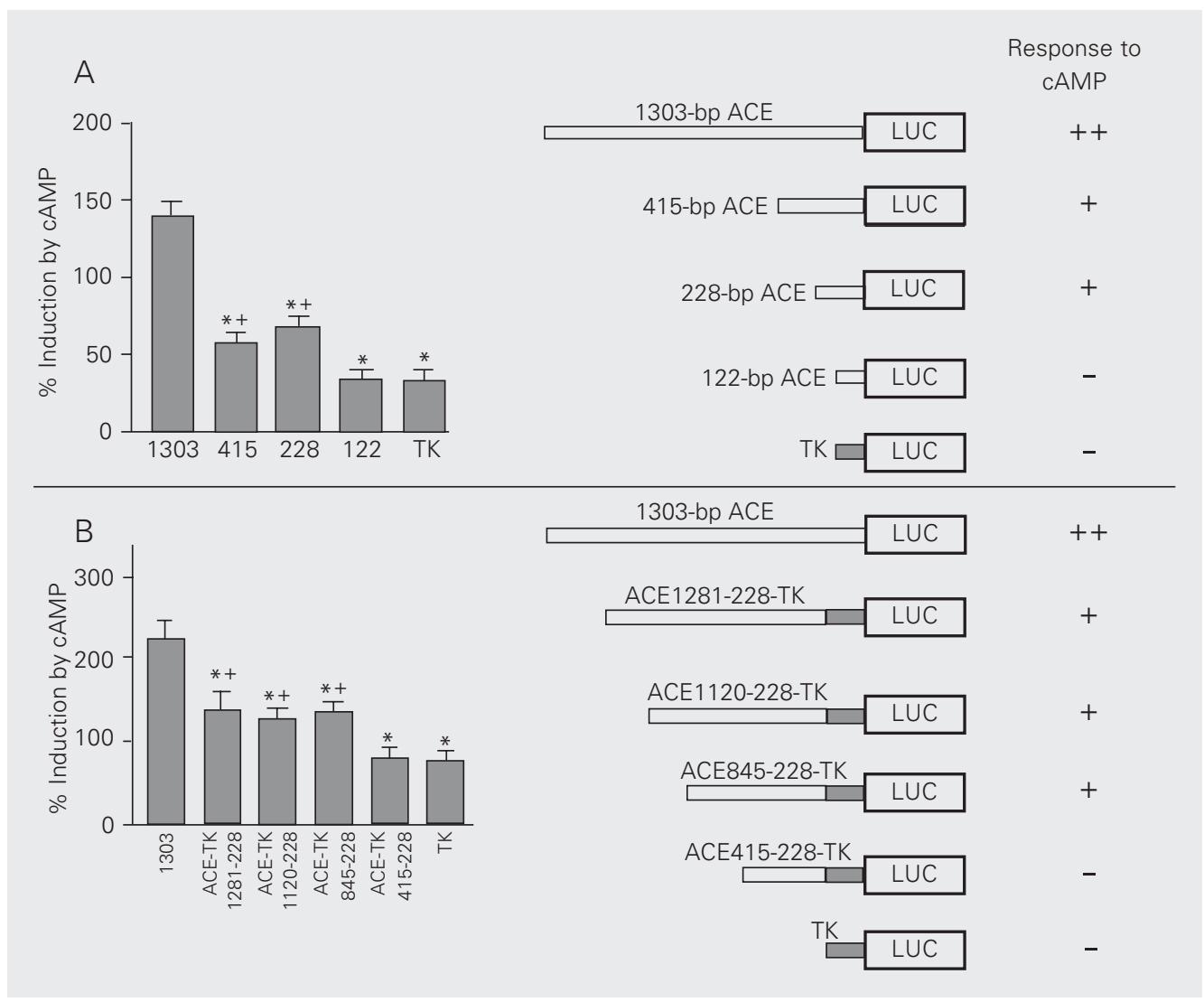

Figure 2. Mapping of the angiotensin converting enzyme (ACE) promoter response to CAMP in an endothelial cell line (REC). A, The wild type 1303-bp ACE promoter, three deletion mutants $(415,228$ and $122 \mathrm{bp})$ and thymidine kinase (TK), a negative control, were transfected in REC to identify promoter sequences involved in the response to cAMP. After $24 \mathrm{~h}$ of transfection cells were serum-starved for $16-24 \mathrm{~h}$ in F12 supplemented with $0.5 \%$ FBS. 8BrcAMP was then administered to the cells at a concentration of $5 \mathrm{mM}$ for $4 \mathrm{~h}$. Luciferase (LUC) activity in cell extracts was normalized for beta galactosidase expression from a control plasmid. Promoter activity is the ratio of luciferase/beta galactoside expression. Data are reported as percent induction compared to control values for each construct. Data are reported as the mean \pm SEM for 4 independent experiments ( $N=4$ in each). $B$, Mapping of cAMP responsiveness in distal ACE promoter sequences. The 1303-bp ACE promoter and hybrid ACE-TK constructs were transfected in REC to narrow the large distal interval containing cAMP-responsive elements between nucleotides 415 and 1303. Fragments 228 to 415, 228 to 845, 228 to 1120 , and 228 to 1281 bp of the ACE promoter were placed upstream from the TK promoter. Transfections and stimulations were performed as above. Data are reported as percent induction compared to control values for each construct. Data are reported as the mean \pm SEM for 5 independent experiments $\left(N=6\right.$ in each). ${ }^{*} P<0.05$ compared to $1303 \mathrm{bp},+P<0.05$ compared to TK 
1303

GATCCTCCTTGTGAACTTCATA GGTACCACCTTCAGAATCCСCTCTTACACCTCCACCAGCACAGCCT 1236 Kpn-I 1281 bp

GCTTCCTCCACAAACACAACAGCTCGCGGATGGGAAATA CACACGTAACCCACAACCCTCAAATTC 1169 TGGGAGCCCAAGCAATGGTGCACCTAAGCTCTCACCAGGTGGCCCTGTGCTAGCCACCTTCCTCCTC 1102 Nhe-l $1120 \mathrm{bp}$

TCTCTACCTCCACCAGCCCCCTAGGACCTCTTCTCT GGGACATGTCACCCAGGCCAGCACAATCTCTC 1034 TGAGTCTCCAGATCACATAA CCTCCTGTATGAACTCTGTCACCACATCTGCCAAGGACAAGGGTCCC 967

CACACCTCTTTGGGGGCACGGTCCTGGTGCTAAGAAGTTAGCCTCCACAGCCTCCTGGTGCCTCAAG 900 AP2

AGGACACGATCCTACCTCCCCCAGGCTTGTCACTTTGAGGGACAGGTGGAGGAGACGAGGAAATGA 834 ++++++ dCRE $845 \mathrm{bp}$

CTGGGAGTGTGGAGACCTGAGGTGACTTGAAGCCGATCTGTCTCAGGGGTAATTTCTCCATTTTTAAA 766

AAGCAGGGGGGTTGCTGTAGTCTCTGAGGCATGTTCTAGCTCTCAAAGCTTGCAACTGCGAGGTTCT 699

AGGGCTTTGTTGGGACAACGCATGATGGTTCTTGCGAGCTGAACAACCGACTGTCACCTGTGTACCC 632

AAAGAGTAGGGCAGATGTGCCAGTGTTCGTGGCCTATTCTATGGCTCCCCCCTCGTAGAGCAGAATA 565

GACCCTATTTCGAGGCATCCCAACAACCCACGCCCAAGGTGAACTGGCCGCTGGCAT CTGCAGTACC 498

TAGCCAAGGCGCCTGTCAGGACACCTAAGCTTCCTGCACCCACGCAGGCGACCCTTCCCTCCATAAC 432

CTGGCAACGTTAAGTCCATCCTGGTGGCACCAGCCAGCCACCCCCGCCCCCTTGTCACTCAAGAGCC 364 Bstx-I 415 bp

CGGAAAGGCTTGGAGGGTTATGCCAAACCTCCTGGGCTCTGTGGCCTGAGAATAAGTGGTGGC GGC 298
AP2
AP2
$+++$

CAGAGGGCACGGTTGGGCCGCAGCACTGTGTTTGC AGCCCGCAGGACCCCTTTCTAGCTTCCTCAGC 231

$++++++++$

PCRE

$+++++++++++$

GAGAGCTCGACCTAACCTCATCCTTCCACCCAAGCTGTTCCCAAGCCCCTCAGGGCTACCCTTGCCC 164 Sac-l 228 bp $* * * * * * * * * * * * * *$

GGGGCGGGCGGGGAATCAGCGGGGCTCGCGCTGCCGGGGACITTGGAGCGAGGAGGAAGCGCGG 100 $122 b p$

AGGGGCGGGGAGGTGGGGGTGTGTCGGGAGGCGGCGGTGACTGGGTTTTATAATCCGCAGGGCGG 35

TCGCGGCGCGGGAGAAGGGGCAGCGCCGCACCGC

Figure 3. Nucleotide sequence of the 1303-bp rat angiotensin converting enzyme (ACE) promoter. Underlined sequences indicate restriction enzyme hydrolysis sites and nucleotide sequences used to construct deletion mutants of the ACE promoter. Sequences underlined with asterisks indicate cAMP-responsive elements (CRE)-like motifs of the ACE promoter. Sequences underlined with plus symbols indicate AP2 motifs of the ACE promoter. 
pCRE and dCRE were replaced with one Bgl-II site and two Bgl-II sites, respectively. As indicated in Figure 5A, mutation of pCRE reduced ACE promoter activity by $33 \%(\mathrm{P}<$ 0.01 ), indicating that $\mathrm{pCRE}$ is a positive cis element controlling basal transcription in REC. Mutation of the two tandem and overlapping CREs within dCRE increased basal ACE promoter activity by $38 \%(\mathrm{P}<0.05)$, indicating that $\mathrm{dCRE}$ is a negative basal cis element in REC. Simultaneous mutation of pCRE and dCRE increased ACE promoter activity by $43 \%(\mathrm{P}<0.01)$, indicating that the combined effect of both ACE CREs is to repress basal transcription in REC.

To test whether pCRE and dCRE are also important for ACE promoter regulation outside the context of endothelial cells we trans- fected the mutated constructs described above in 293 cells. In contrast to REC, mutation in either pCRE or dCRE greatly increased promoter activity, indicating that both sequences exert a negative role in the 293 context. As expected, simultaneous mutation of pCRE and dCRE further increased promoter activity in 293 (Figure 5B).

Since mapping of functional cAMP-responsive units in the ACE promoter was consistent with the position of both pCRE and dCRE, we tested whether individual or combined mutations in these motifs would affect cAMP responsiveness of the 1303-bp ACE promoter. Surprisingly, neither pCRE, dCRE, or double pCRE/dCRE mutants affected the cAMP responsiveness of the ACE promoter in REC or in 293 (Figure 5C,D).
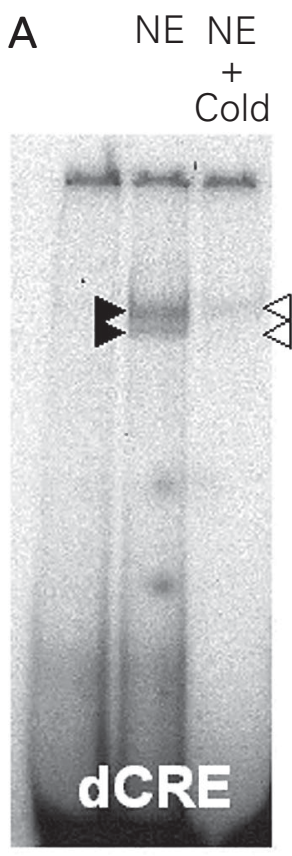
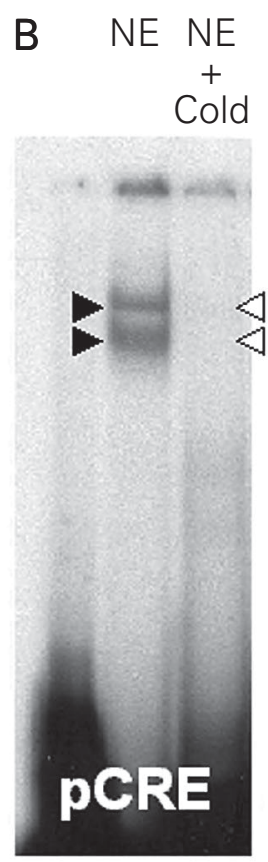

C

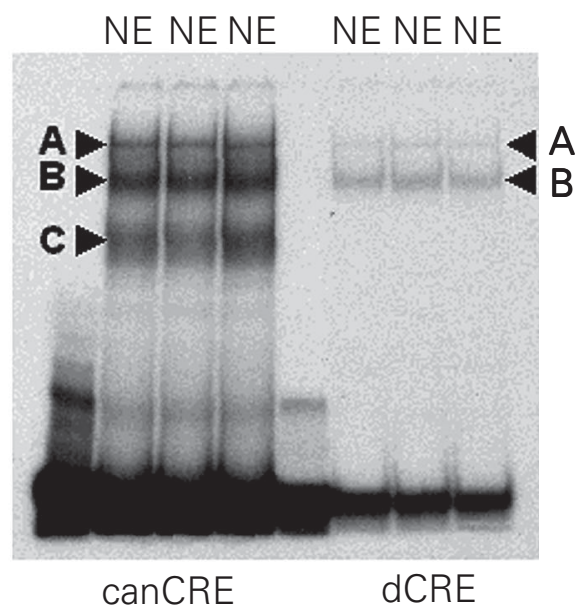

Figure 4. Gel retardation analysis of the cAMP-responsive elements pCRE and dCRE. Nuclear extracts of an endothelial cell line (REC) were combined with ${ }^{32}$ P-labelled duplex oligonucleotides containing sequences of ACE CRE-like elements. Binding to the labeled oligonucleotide was reduced with addition of a 50-fold excess of an unlabeled duplex oligonucleotide containing the canonical CRE sequence. A, Nuclear extracts of REC produce two dCRE-retarded bands whose intensity is reduced by addition of a 50 -fold excess of an unlabeled canonical CRE probe. $B$, Nuclear extracts of REC produce two pCRE-retarded bands that are also reduced by addition of a 50-fold molar excess of the unlabeled canonical CRE probe. $C$, Triplicate binding reactions for canonical CRE (canCRE) and dCRE suggest that dCRE binds with lower affinity to two out of three protein complexes binding the canonical CRE. NE = nuclear extract; Cold $=50 \times$ unlabeled canonical CRE nucleotide. Black arrowheads indicate protein complexes (bands) binding to the labeled oligonucleotide. White arrowheads indicate bands whose intensities are reduced by excess of cold oligonucleotides ( $A$ and $B$ ). 
This indicates that the 1303-bp ACE promoter can respond effectively to cAMP even in the absence of CRE-like sequences that regulate its basal expression.

Trans regulation of the somatic ACE promoter by ATF-2 and its heterodimerization partner c-Jun

The presence of CRE-like sequences of the ACE promoter (Figure 4) suggested that the ACE gene is a target for regulation by signaling pathways distinct from the classic cAMP pathway, such as those involving the stress-activated JNK or P38 kinases (22). To establish whether the ACE promoter is regulated by such pathways in endothelial cells we transfected the 1303-bp ACE promoter along with expression vectors for ATF-2 and for its heterodimerization partner, c-Jun. Cotransfection with ATF-2 reduced luciferase expression from the 1303 ACE promoter by $30 \%$. Further decreases in luciferase expression were obtained when ATF-2 was co-

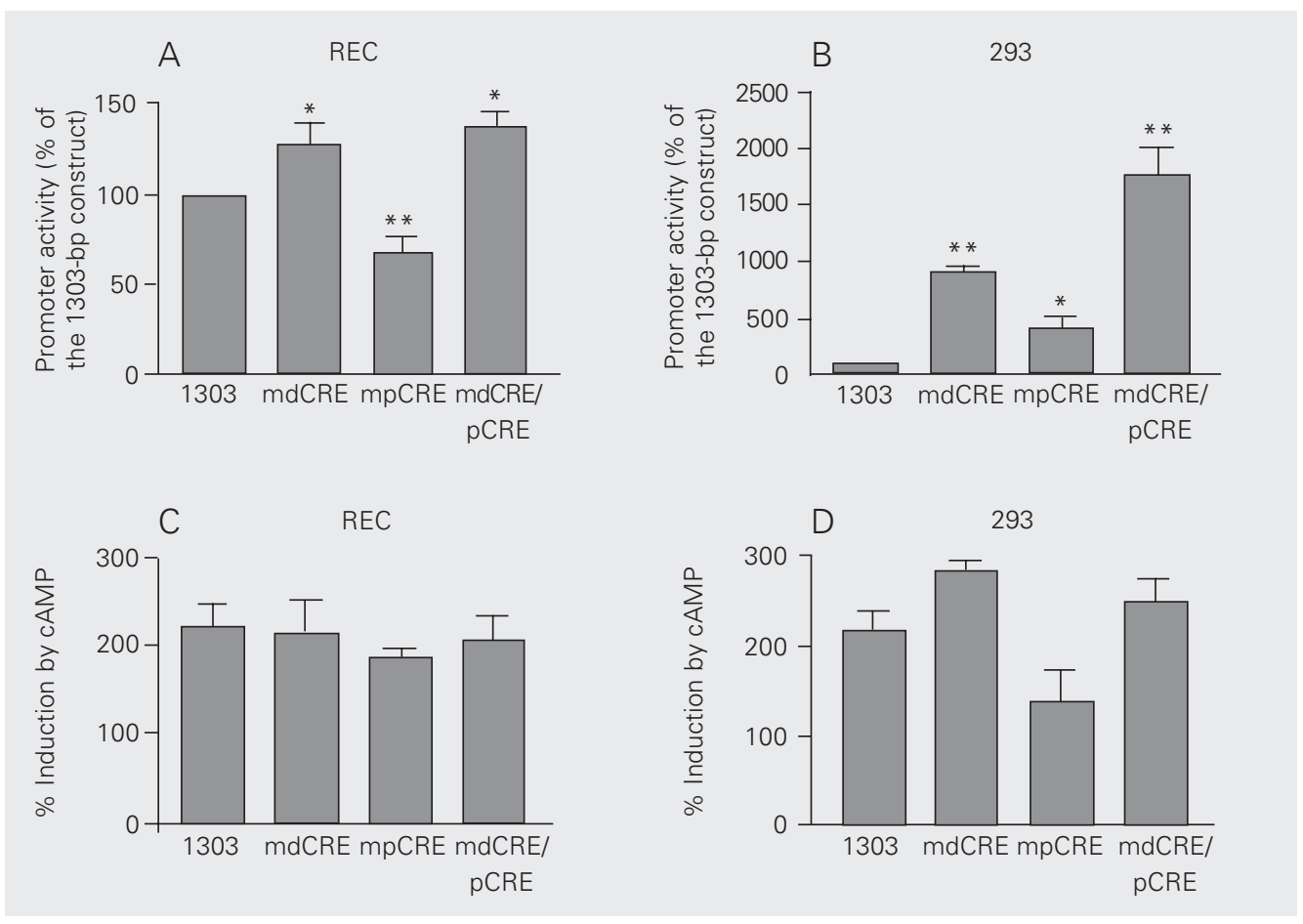

Figure 5. Role of the cAMP-responsive elements dCRE and pCRE in basal and CAMP-induced transcription in endothelial and renal cells. A, Basal transcription in endothelial cells. The wild type 1303-bp promoter and mutants harboring Bgl-II restriction sites in place of dCRE (mdCRE), pCRE (mpCRE) and in both CRE-like sequences (mdCRE/pCRE) were transfected in rabbit endothelial cell (REC). Cells were harvested $24 \mathrm{~h}$ after transfection. Luciferase activity in cell extracts was normalized to beta galactosidase expression from a control plasmid. Promoter activity is the ratio of luciferase/beta galactoside expression and is reported as percent of the activity in the 1303-bp angiotensin converting enzyme (ACE) promoter. Data are reported as the mean \pm SEM for 5 independent experiments ( $N=6$ in each). $B$, Basal transcription in renal cells: 293 cells were transfected with the same promoter constructs as in $A$ and results were analyzed and reported as described for panel $A$. $C$, cAMPinduced transcription in endothelial cells. The wild type 1303-bp ACE promoter and the mutants harboring substitutions in CRE-like sequences were transfected in an endothelial cell line (REC) to identify promoter sequences involved in the response to cAMP. After $24 \mathrm{~h}$ of transfection, cells were serum-starved for 16-24 $\mathrm{h}$ on F12 supplemented with $0.5 \%$ FBS and then stimulated with $5 \mathrm{mM} 8$ BrcAMP for $4 \mathrm{~h}$. Data are reported as percent induction compared to control values for each construct. Data are reported as the mean \pm SEM for 4 independent experiments ( $N=6$ in each). $D$, cAMP-induced transcription in renal cells. 293 cells were transfected with the same promoter constructs as in $C$ and results were analyzed and reported as in panel $A$. ${ }^{*} P<0.05 ;{ }^{*} P<0.01$ compared to the 1303-bp ACE promoter (one-way analysis of variance and post hoc Tukey test). 
expressed with c-Jun. In contrast, co-transfection with c-Jun alone did not change luciferase expression by the 1303 ACE promoter. Thus, these results suggest that repression of the 1303 ACE promoter by ATF2 is consistent with the ATF-2 protein acting as a homodimer or heterodimer together with c-Jun (Figure 6).

\section{BrcAMP activates CRE rather than AP2 pathways in REC}

Mutational analysis of dCRE and pCRE indicated that both motifs are dispensable for cAMP induction of the 1303-bp ACE

Figure 6. ATF-2 inhibits the 1303bp angiotensin converting enzyme (ACE) promoter in an endothelial cell line (REC). The 1303-bp ACE promoter was cotransfected with expression vectors for transcription factors ATF2 and c-Jun, separately or in combination. Cells were harvested $24 \mathrm{~h}$ after transfection. Luciferase activity in cell extracts was normalized for beta galactosidase expression from a control plasmid. Promoter activ-

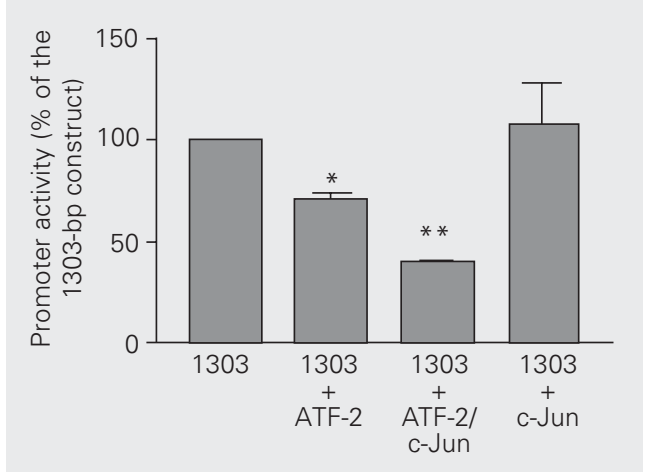

ity is the ratio of luciferase/beta galactoside expression and is reported as percent of the activity of the 1303-bp ACE promoter. Data are reported as the mean \pm SEM for 3 independent experiments $(N=6)$. ${ }^{*} P<0.05$ compared to $1303 \mathrm{bp},{ }^{*} P<0.01$ compared to 1303 bp (one-way analysis of variance and post hoc Tukey test).

Figure 7. cAMP regulation of the 1303-bp angiotensin converting enzyme (ACE) promoter in an endothelial cell line (REC). The 1303-bp ACE promoter, positive controls for stimulation of the cAMP-responsive elements (CRETK) and AP2 (AP2LUC) pathways and a negative control (TATA) were co-transfected with expression vectors for AP2 when appropriate. After $24 \mathrm{~h}$ of transfection, cells were serumstarved for 16-24 h on F12

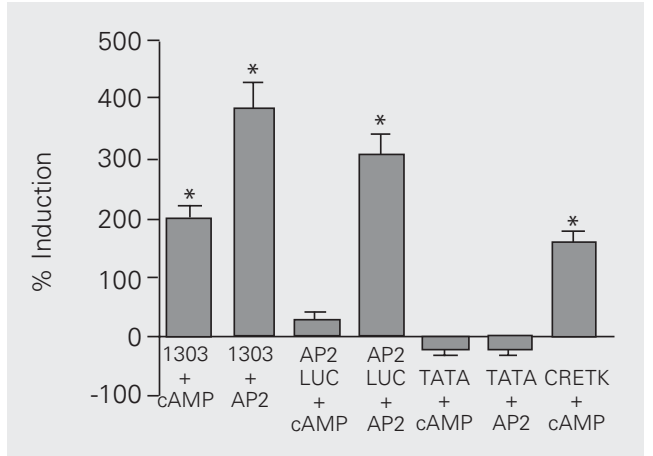

supplemented with $0.5 \%$ FBS. Transfected cells were then stimulated with 5 mM 8BrcAMP for $4 \mathrm{~h}$ when indicated. Luciferase (LUC) activity in cell extracts was normalized for beta galactosidase expression by a control plasmid. Promoter activity is the ratio of luciferase/ beta galactoside expression and is reported as percent induction compared to control values for each construct. Data are reported as the mean \pm SEM for 4 independent experiments $(N=6)$. ${ }^{*} P<0.01$ indicates statistically significant induction from unstimulated controls (one-way analysis of variance and post hoc Tukey test). promoter (Figure 5C,D). These results suggest that ACE promoter activation by cAMP is either due to extremely divergent CRElike sequences, or alternatively to the potential AP2 sites present in the 1303-bp promoter (Figure 3). In Figure 7 we compared the relative efficiency of the AP2 and CRE pathways in the transduction of the cAMP signal. In REC, 8BrcAMP efficiently induced CRETK, the positive control for the CRE pathway, but failed to affect luciferase expression by AP2LUC, the positive control for the AP2 pathway. Co-transfection of an AP2 expression vector demonstrated that the AP2LUC construct is fully functional. Therefore, our data for REC suggest that induction of cAMP signaling does not lead to activation of the AP2 pathways.

\section{Discussion}

In the present study we used a transient transfection strategy in REC and 293 cells to assess the role of CRE-like motifs of the rat somatic ACE promoter in basal as well as in cAMP-stimulated transcription. Major findings include 1) description of a distal CRE (dCRE), conserved between rats, mice and humans, as a negative basal cis element in REC and 293; 2) description of a non-conserved proximal CRE (pCRE) as a positive basal cis element in REC and as a negative basal cis element in 293; 3) characterization of ATF-2 as a potential regulator of ACE gene expression; 4) mapping of cAMP responsiveness to two independent domains within the 1303-bp ACE promoter; 5) assessment of the relative roles of CRE versus AP2 pathways in the response to cAMP in REC.

Functional analysis of the 1303-bp ACE promoter identified two regions containing regulators of basal and cAMP-induced expression: a proximal one between 122 and 288 bp and a distal one between 415 and 1303 bp. Screening for CRE-like sequences in these regions revealed pCRE, between 209 and 222 bp and dCRE, between 801 and 
819 bp. pCRE and dCRE represent the closest approximations to a canonical CRE within the 1303-bp ACE promoter. Although pCRE and dCRE contain substitutions at the CRE core binding site (CGTCA), inspection of labeled bands in the gelshifts of Figure 5C suggests that pCRE and dCRE bind two out of three protein complexes binding to the canonical CRE in REC nuclear extracts, albeit at lower affinity. More importantly, binding to $\mathrm{pCRE}$ and dCRE can be displaced by competition with cold oligonucleotides containing the canonical CRE. Thus, pCRE and dCRE share many properties with the canonical CRE and therefore must be classified as CRE-like sequences.

We established the roles of pCRE and dCRE in basal transcription by mutation analysis. As shown in Figure 5A, mutation of pCRE reduces basal activity by about half, suggesting that it may participate in the increase in luciferase expression observed from the 122- to the 228-bp promoter construct. Conversely, mutation of dCRE produces a small but significant increase in basal activity. As such, dCRE probably contributes to the inhibition of basal expression observed from the 415- to the 1303-bp promoter construct. Combined mutation of pCRE and dCRE produced a net increase in basal activity. This indicates that pCRE and dCRE must interact to produce a net inhibitory regulation different from the simple addition of their individual effects in REC.

The 1303-bp rat ACE promoter responds to the activation of CRE and AP2 signaling pathways. However, in REC, 8BrcAMP induces the CRE, but not the AP2 reporter. This is in contrast to other systems where CRE and AP2 motifs transduce the cAMP signal either separately or in concert (13). As such, the resistance of the AP2 reporter to stimulation by cAMP is inconsistent with a major role of AP2 signaling in the REC response to cAMP. Moreover, all ACE AP2 sites, located between 228 and $415 \mathrm{bp}$ as well as between 845 and 1120 bp (Figure 3), fell outside the two domains of cAMP responsiveness that we mapped (Figure 2). Thus, our data suggest that, in REC, the bulk of ACE promoter activation in response to cAMP is due to CRE mechanisms. It remains to be established whether the refractoriness of the AP2 pathway to cAMP stimulation in REC is shared by most endothelial cell types, or if it is a peculiar feature of the REC line.

Because individual or combined mutation of pCRE and dCRE did not affect induction by 8BrcAMP, the CREs mediating activation of the ACE promoter by cAMP remains unaccounted for. Due to the pronounced divergence of the remaining candidates from the CRE consensus it is likely that identification of these putative elements will require the use of linker-scanning strategies (23).

The arrangement of CRE-like sequences in the ACE promoter is compatible with those of numerous promoters that display multiple CRE-like motifs scattered in their sequences. CRE-like motifs in these promoters are usually multimers or single elements closely associated with other motifs and located in separated domains $(24,25)$. Among those CRElike sequences, some have effects on basal or on cAMP-stimulated transcription or on both. These CRE-like motifs play either positive or negative roles in basal transcription $(26,27)$ and display redundant roles in the response to cAMP $(24,28)$. In the present study we have shown that dCRE, a motif conserved between rats, mice and humans, is a negative cis regulator of $\mathrm{ACE}$ gene basal expression. This finding is consistent with work showing that conserved non-canonical CREs are regulators of basal transcription in the promoters of cystic fibrosis transmembrane conductance genes from 8 mammalian species (29) as well as in the promoter for the testicular ACE isozyme $(30,31)$.

In the present study we used rabbit endothelial and human renal cell lines to study the rat ACE promoter. This approach can be criticized on the grounds that promoter regulation can be species-specific. However, 
mechanisms of gene regulation have been extensively modeled in heterologous contexts and ACE regulation by cAMP, in particular, is a conserved feature among mammals (for a review, see Ref. 10). REC is a cell line that maintains stable endothelial features such as uptake of 1,19-dioctadecyl3', 3', 3', 3'-tetramethylindocarbocyanine perchlorate-labeled acetylated LDL and ACE gene expression (10). Thus, REC compares favorably with primary cultures of endothelial cells, which display limited growth characteristics, do not sustain expression of endothelial markers and are refractory to transfection in mammals (10).

The advantages and disadvantages of the heterologous approach are represented in our study. As shown in Figure 5, dCRE, which is conserved between rats, mice and men, is an inhibitory element in both rabbit and human cell lines, suggesting that this is its role in the native setting. In contrast, pCRE has a positive role in rabbits and a negative role in a human cell line, a finding which is consistent with the lack of evolutionary conservation in this motif between the aforementioned species. While the actual role of pCRE in its native setting cannot be established by our experiments, we believe nonetheless that the differences in the regulatory potential of pCRE can be easily accounted for by quantitative and qualitative cell-specific differences in transcription factor content. This scenario is typical of the evolution of cis regulatory sequences, when one or more redundant units can diverge without risking basic promoter functions (32).

What is then the relevance of pCRE and dCRE for ACE regulation in vivo? In experiments that will not be documented here we generated transgenic mice for the wild type 1303-bp ACE promoter as well as for the double mutant mdCRE/pCRE. While the wild type 1303-bp promoter was not enough to drive reporter expression in kidneys, lungs and ovaries, the double mutant induced strong expression in all of these organs (Anéas I, unpublished results). These results, taken together with our demonstration that $\mathrm{pCRE}$ and dCRE interact to repress basal transcription from the ACE promoter in endothelial and renal cell types, suggest that both CRElike sequences are important targets for tissue-specific regulation of the ACE gene.

Our data for REC and 293 cells suggest that the 1303-bp ACE promoter has a potential for negative regulation by CRE-like sequences that could bind transcription factors such as ATF-2, which conveys signaling inputs from pathways other the cAMP pathway (22). To test whether ATF-2 negatively regulates the ACE promoter in endothelial cells we co-transfected ATF-2 and c-Jun expression vectors with the 1303-bp ACE promoter and showed that, indeed, ATF-2 alone, or in combination with c-Jun, negatively regulates ACE promoter activity. Our description of ACE promoter regulation by ATF-2 indicates a potential pathway of somatic ACE gene regulation in endothelial cells. ATF-2/c-Jun heterodimers are phosphorylated by p38 stress kinases activated by UV radiation, bacterial DNA, hyperosmolar stress, and oxidative stress (22). A great deal of information has been accumulated implicating stress-activated kinases, ATF-2 and c-Jun in the response to myocardial ischemia-reperfusion as well as in ischemic preconditioning. As such, our data suggest that negative regulation of ACE gene expression in the endothelium may be part of the mechanisms that protect the myocardium after a bout of ischemia-reperfusion. This raises the interesting possibility that ACE inhibitors are favorable in chronic myocardial ischemia because they mimic an endogenous genetic program of myocardial protection triggered in endothelial or in other cell types by ischemia-reperfusion. In summary, the link between ACE regulation, ATF-2/c-Jun and stress-activated kinases opens several interesting possibilities to be explored in cardiovascular (6) as well as in chronic inflammatory diseases (7) in which ACE plays a role. 


\section{References}

1. Friedland J, Setton C \& Silverstein E (1977). Angiotensin converting enzyme: induction by steroids in rabbit alveolar macrophages in culture. Science, 197: 64-65.

2. Krulewitz AH, Baur WE \& Fanburg BL (1984). Hormonal influence on endothelial cell angiotensin-converting enzyme activity. American Journal of Physiology, 247: C163-C168.

3. Dasarathy $Y$, Lanzillo JJ \& Fanburg BL (1982). Stimulation of bovine pulmonary artery endothelial cell ACE by dexamethasone: involvement of steroid receptors. American Journal of Physiology, 263: H645-H649.

4. Dasarathy Y \& Fanburg BL (1989). Calcium ionophore A23187 elevates angiotensin-converting enzyme in cultured bovine endothelial cells. Biochimica et Biophysica Acta, 1010: 16-19.

5. Fishel RS, Thourani V, Eisenberg SJ, Shai SY, Corson MA, Nabel EG, Bernstein KE \& Berk BC (1995). Fibroblast growth factor stimulates angiotensin converting enzyme expression in vascular smooth muscle cells. Possible mediator of the response to vascular injury. Journal of Clinical Investigation, 95: 377-387.

6. Kyriakis JM \& Avruch J (2001). Mammalian mitogen-activated protein kinase signal transduction pathways activated by stress and inflammation. Physiological Reviews, 81: 807-869.

7. Weinstock JV (1986). The significance of angiotensin I converting enzyme in granulomatous inflammation. Functions of ACE in granulomas. Sarcoidosis, 3: 19-26.

8. Lloyd CJ, Cary DA \& Mendelsohn FAO (1987). Angiotensin converting enzyme induction by cyclic AMP and analogues in cultured endothelial cells. Molecular and Cellular Endocrinology, 52: 219225.

9. Dasarathy $Y$ \& Fanburg BL (1988). Elevation of angiotensin converting enzyme by 3-isobutyl-1-methylxanthine in cultured endothelial cells: a possible role for calmodulin. Journal of Cellular Physiology, 137: 179-184.

10. Xavier-Neto J, Pereira AC, Junqueira ML, Carmona $R$ \& Krieger JE (1999). Rat angiotensin-converting enzyme promoter regulation by beta-adrenergics and cAMP in endothelium. Hypertension, 34: 3138.

11. Montminy MR (2001). Transcriptional regulation by the phosphorylation-dependent factor CREB. Nature Reviews. Molecular Cell Biology, 2: 599-609.

12. Roesler WJ (2000). What is a cAMP response unit? Molecular and Cellular Endocrinology, 162: 1-7.

13. Gao B, Chen J, Johnson C \& Kunos G (1997). Both the cyclic AMP response element and the activator protein 2 binding site mediate basal and cyclic AMP-induced transcription from the dominant promoter of the rat alpha 1B-adrenergic receptor gene in DDT1MF-2 cells. Molecular Pharmacology, 52: 1019-1026.

14. Xavier-Neto J, Pereira AC, Motoyama AH \& Krieger JE (1998). A luciferase-engineered cell line for study of cAMP regulation in endothelial cells. American Journal of Physiology, 275: C75-C81.

15. Matsuda S, Maekawa T \& Ishii S (1991). Identification of the functional domains of the transcriptional regulator CRE-BP1. Journal of Biological Chemistry, 266: 18188-18193.

16. Williams T \& Tjian R (1991). Analysis of the DNA-binding and activation properties of the human transcription factor AP-2. Genes and Development, 5: 670-682.

17. Doucas V, Spyrou G \& Yaniv M (1991). Unregulated expression of c-
Jun or c-Fos proteins but not Jun D inhibits oestrogen receptor activity in human breast cancer derived cells. EMBO Journal, 10: 2237-2245.

18. Buonassisi V \& Venter JC (1976). Hormone and neurotransmitter receptors in an established vascular endothelial cell line. Proceedings of the National Academy of Sciences, USA, 73: 1612-1616.

19. Graham FL \& van der Eb AJ (1973). A new technique for the assay of infectivity of human adenovirus 5 DNA. Virology, 52: 456-459.

20. Ribeiro RC, Apriletti JW, Yen PM, Chin WW \& Baxter JD (1945). Heterodimerization and deoxyribonucleic acid-binding properties of a retinoid X receptor-related factor. Endocrinology, 135: 2076-2085.

21. Krieger JE, Junqueira ML \& Dzau VJ (1994). Characterization of the promoter from rat angiotensin-converting enzyme gene. Circulation, 90: 130 (Abstract).

22. Johnson GL \& Lapadat R (2002). Mitogen-activated protein kinase pathways mediated by ERK, JNK, and p38 protein kinases. Science, 298: 1911-1912.

23. Gustin K \& Burk RD (2000). PCR-directed linker scanning mutagenesis. Methods in Molecular Biology, 130: 85-90.

24. Fisch TM, Prywes R, Simon MC \& Roeder RG (1989). Multiple sequence elements in the c-fos promoter mediate induction by cAMP. Genes and Development, 3: 198-211.

25. Soubt MK, Marksitzer R, Menoud PA \& Nagamine $Y$ (1998). Role of tissue-specific transcription factor LFB3 in a cyclic AMP-responsive enhancer of the urokinase-type plasminogen activator gene in LLCPK1 cells. Molecular and Cellular Biology, 18: 4698-4706.

26. Jungmann RA, Huang D \& Tian D (1998). Regulation of LDH-A gene expression by transcriptional and posttranscriptional signal transduction mechanisms. Journal of Experimental Zoology, 282: 188195.

27. Murasawa S, Matsubara H, Kijima K, Maruyama K, Ohkubo N, Mori Y, Iwasaka T \& Inada M (1996). Down-regulation by cAMP of angiotensin II type 2 receptor gene expression in PC12 cells. Hypertension Research, 19: 271-279.

28. Giono LE, Varone CL \& Canepa ET (2001). 5-Aminolaevulinate synthase gene promoter contains two cAMP-response element (CRE)like sites that confer positive and negative responsiveness to CREbinding protein (CREB). Biochemical Journal, 353: 307-316.

29. Vuillaumier S, Dixmeras I, Messai H, Lapoumeroulie C, Lallemand D, Gekas J, Chehab FF, Perret C, Elion J \& Denamur E (1997). Cross-species characterization of the promoter region of the cystic fibrosis transmembrane conductance regulator gene reveals multiple levels of regulation. Biochemical Journal, 327: 651-662.

30. Esther Jr CR, Semeniuk D, Marino EM, Zhou Y, Overbeek PA \& Bernstein KE (1997). Expression of testis angiotensin-converting enzyme is mediated by a cyclic AMP responsive element. Laboratory Investigation, 77: 483-488.

31. Kessler SP, Rowe TM, Blendy JA, Erickson RP \& Sen GC (1998). A cyclic AMP response element in the angiotensin-converting enzyme gene and the transcription factor CREM are required for transcription of the mRNA for the testicular isozyme. Journal of Biological Chemistry, 273: 9971-9975.

32. Hancock JM, Shaw PJ, Bonneton F \& Dover GA (1999). High sequence turnover in the regulatory regions of the developmental gene hunchback in insects. Molecular Biology and Evolution, 16: 253-265. 